\title{
Dimensões do desenvolvimento humano: o caso da região de Ribeirão Preto, SP
}

Dimensions of human development: the case of Ribeirão Preto region, SP

\section{Dimensions du développement humain: le cas de la région de Ribeirão Preto, SP}

Dimensiones del desarrollo humano: el caso de la región de Ribeirão Preto, SP

\author{
André Cavalcante da Silva Batalhão ${ }^{1}$ \\ Gláucia Aparecida Prates² \\ Denílson Teixeira ${ }^{3}$ \\ Emiliano Lobo de Godoi ${ }^{3}$
}

Recebido em 24/08/2017; revisado e aprovado em 13/09/2017; aceito em 24/09/2017

DOI: http://dx.doi.org/10.20435/inter.v0i0.1692

\begin{abstract}
Resumo: O objetivo principal da pesquisa foi analisar o nível de desenvolvimento humano dos 25 municípios da Região Administrativa de Ribeirão Preto, Estado de São Paulo, Brasil, usando o Índice de Desenvolvimento Humano Municipal, e apontar fragilidades e potencialidades desse índice. A metodologia possui característica exploratória, descritiva e crítica, baseada em dados secundários. O município de Serra Azul apresentou a maioria dos indicadores críticos que compõem o índice.
\end{abstract}

Palavras-chave: Índice de Desenvolvimento Humano Municipal; desenvolvimento regional; desenvolvimento local; região de Ribeirão Preto.

\begin{abstract}
The aim of research was to analyze the level of human development of the 25 municipalities in the Administrative Region of Ribeirão Preto, São Paulo's State, Brazil, using Municipal Human Development Index, and to inform weaknesses and potentialities of the tool. The methodology has exploratory and descriptive characteristics, based on secondary data. The municipality of Serra Azul presented most of the critical indicators that compose the index.
\end{abstract}

Keywords: Municipal Human Development Index; regional development; local development; Ribeirão Preto region.

Résumé: L'objectif principal de la recherche était d'analyser le niveau de développement humain des 25 municipalités de la Région administrative de Ribeirão Preto, État de São Paulo, au Brésil, en utilisant l'indice municipal de développement humain et souligner les faiblesses et les potentialités de cet indice. La méthodologie présente des caractéristiques exploratoires, descriptives et critiques, basées sur des données secondaires. La municipalité de Serra Azul a présenté la plupart des indicateurs critiques qui composent l'indice.

Mots-clés: Indice de Développement Humain Municipal; developpement regional; developpement local; région de Ribeirão Preto.

Resumen: El objetivo principal de la investigación fue analizar el nivel de desarrollo humano de los 25 municipios de la Región Administrativa de Ribeirão Preto, Estado de São Paulo, Brasil, usando el Índice de Desarrollo Humano Municipal, y señalar fragilidades y potencialidades de este índice. La metodología posee característica exploratoria, descriptiva y crítica, basada en datos secundarios. El municipio de Serra Azul presentó la mayoría de los indicadores críticos que componen el índice.

Palabras clave: Índice de Desarrollo Humano Municipal; desarrollo regional; desarrollo local; región de Ribeirão Preto.

\footnotetext{
${ }^{1}$ Universidade Federal de Goiás (UFG), Goiânia, Goiás, Brasil. Universidade Nova de Lisboa, Caparica, Portugal.

${ }^{2}$ Universidade Estadual Paulista (Unesp), Itapeva, São Paulo, Brasil.

${ }^{3}$ Universidade Federal de Goiás (UFG), Goiânia, Goiás, Brasil.
} 


\section{INTRODUÇÃO}

No mundo contemporâneo, a sociedade tem constantemente se redefinido, e isso influencia os padrões de desenvolvimento e suas próprias definições. Conceitos de desenvolvimento e ideários de ampliação de promoção de qualidade de vida, logicamente deveriam acompanhar o delineamento de uma crônica incompletude social existente, que compromete outras esferas do desenvolvimento humano, principalmente em consideração à aceitação da necessidade de diálogo entre eixos temáticos que vão além do social e econômico. A expansão, ou até mesmo, a manutenção de políticas públicas, devem incorporar em seu corpo, expectativas e demandas que relativizem as influências atuais das sociedades, e as novas dinâmicas de desenvolvimento. Deve-se enfatizar, também, que o desenvolvimento humano inclui expandir o bem-estar de todas as pessoas e aumentar as possibilidades de escolha individual (PLANO DAS NAÇÕES UNIDAS PARA O DESENVOLVIMENTO [PNUD], 2014a, 2014b), e pode ser definido como o processo de expansão da capacidade das pessoas para realizarem suas atividades, sem restrições (SEN, 2000). A abordagem do desenvolvimento humano apareceu como um meio para realocar o ser humano no centro das ações relacionadas à política, à economia e à sociedade, de tal forma que a preocupação central não é mais sobre o quanto está sendo produzido e, sim, como tudo isso afeta e influencia a qualidade de vida das pessoas (GOR; GITAU, 2010).

O caráter interdisciplinar da reflexão sobre o desenvolvimento contribuiu para ampliar e aprofundar a consciência crítica do homem contemporâneo. A aspiração para a reflexão sobre o desenvolvimento advém da realidade social, a partir da identificação das entidades envolvidas que assumem novas formas. Essa realidade social se desenvolve como algo estruturado, estabelecendo-se como um processo (FURTADO, 2000). Nesse contexto, o subdesenvolvimento não pode ser estudado como uma fase do processo de desenvolvimento (FURTADO, 2003). O subdesenvolvimento requer um esforço de teorização autônomo e, portanto, não deve ser considerado apenas uma etapa pela qual tenham, necessariamente, passado as economias que já alcançaram um grau superior de desenvolvimento (FURTADO, 2009). Dessa maneira, o desenvolvimento e o subdesenvolvimento devem ser considerados como dois aspectos de um mesmo processo histórico, ou seja, não irredutíveis a esquemas formalizáveis (FURTADO, 2003; 1984).

A multiplicidade do desenvolvimento local é resultante de múltiplas ações convergentes e complementares, que podem quebrar o elo de dependência e inoperância do subdesenvolvimento de localidades afastadas do centro e promover uma mudança social no território. É necessário não se limitar ao enfoque estritamente econômico e contemplar outras faces do desenvolvimento humano, para promover uma integração regional e reduzir a dependência de transferência de rendas originárias de outros territórios (BUARQUE, 2008). Diante das desigualdades regionais, a multiplicidade das relações socioespaciais está cada vez mais voltada para fora da linha do espaço regional, promovendo influências e um ordenamento territorial articulado com espaços mais distantes, fortalecendo uma articulação extensiva do planejamento local-regional.

Pela perspectiva geopolítica, as desigualdades podem ser entendidas como uma característica inerente ao desenvolvimento, em que, em fases subsequentes do próprio processo de desenvolvimento, seria garantida a eliminação dessas desigualdades, aumentando a mobilidade dos fatores de produção e criando melhores condições de acumulação dos excedentes econômicos (FURTADO, 1967). Já pela perspectiva normativa, a Política Nacional de Desenvolvimento Regional (PNDR) tem o papel de orientar os programas e ações federais no território nacional com estratégias que devem convergir com a inclusão social, de produtividade, sustentabilidade 
ambiental e competitividade econômica. Seu principal objetivo é reduzir as desigualdades de nível de vida entre as regiões brasileiras e promover a equidade no acesso a oportunidades de desenvolvimento (BRASIL, 2011).

No período subsequente à Segunda Guerra Mundial, houve uma tomada de consciência sobre o atraso econômico em que vivia a grande maioria da humanidade. Com isso, indicadores como mortalidade infantil, incidência de enfermidades contagiosas, grau de alfabetização e outros logo foram lembrados. Isso contribuiu para dar início às ideias de desenvolvimento, bem-estar social e modernização, enfim, tudo que sugeria acesso às formas de vida criadas pela civilização industrial (FURTADO, 2000).

O Índice de Desenvolvimento Humano (IDH) foi introduzido em 1990 com o objetivo de medir o desenvolvimento em diferentes comunidades considerando três dimensões básicas: expectativa de vida, educação e Produto Interno Bruto (PIB) per capita (ANAND; SEN, 1994; SAGAR, NAJAM, 1998; SLAVOMIR, 2016, MAZOUCH; VLTAVSKÁ; STAŇEK, 2016). Esta métrica baseia-se na ideia básica de que o pré-requisito para alcançar uma vida melhor, além de ter um bom nível de renda, é o florescimento e o desenvolvimento de talentos e capacidades humanas.

A tentativa de conhecer o complexo que compõe a realidade socioeconômica e cultural das nações do planeta é uma problemática a ser compreendida. Tratando de classificações quantitativas ou qualitativas, existem muitas abordagens possíveis, por conseguinte, são inúmeras as formas de classificar e construir arranjos metodológicos que tenham como objetivo criar um quadro síntese de realidade (ORSI; GODOY, 2010). A determinação do efeito local do IDH se faz relevante para o tratamento apropriado das dimensões consideradas nesse índice, em que a preocupação em escala local é um grande detalhe. Quanto mais estudos com enfoque específico nas dimensões de desenvolvimento humano, mais aparentes se tornam os problemas dimensionais identificados nesse âmbito, possibilitando a sugestão de novas abordagens na gestão pública municipal e em suas organizações funcionais envolvidas e integradas nesse processo.

O Índice de Desenvolvimento Humano Municipal (IDH-M) é uma proposta que proporciona ao usuário a visualização de retratos situacionais, a partir de variáveis que o compõem, em escala local, agregando à sua essência o efeito comparativo. Para os governos municipal, estadual e federal, as informações provenientes da ferramenta IDH-M, servem como um instrumento de gestão auxiliar (papel complementar) a ser considerado na formulação de estratégicas políticas e políticas públicas, coordenando o curso do processo decisório, a fim de evitar conflitos e defasagens no modelo gerencial (RAHAYU; RAVIYANTI; MAHARDIKA, 2016).

De acordo com Plano das Nações Unidas para o Desenvolvimento (PNUD, 2014a), foi observado o uso crescente do IDH-M como critério de elegibilidade de municípios para o recebimento de programas, recursos ou políticas públicas, em que o índice também vem sendo utilizado na elaboração de projetos de lei e como critério na concessão de financiamentos públicos. Ainda no âmbito municipal, o índice também provocou ações e discussões com o objetivo de inspirar e fortalecer a governança local, com audiências públicas, encontros de prefeitos, sessões parlamentares especiais, dentre outros. No âmbito estadual, se realça o exemplo dos Estados de Minas Gerais e Acre, que balizaram suas ações a partir do índice, em um programa de combate à pobreza e num projeto de saneamento básico, respectivamente. O governo federal também utilizou o IDH-M como critério em programas sociais, como por exemplo os programas Mais Médicos e Mais Professores, em que o índice serviu como critério de distribuição dos profissionais desses programas nos municípios do Brasil (PNUD, 2014a). 
A discussão acadêmica se faz relevante na abordagem e discussão sobre o tema desenvolvimento humano, pois tem a pretensão de oferecer uma base sólida para uma análise experimental de possibilidades, e assim ser entendida como um subsídio para uma discussão mais ampla, envolvendo amplas parcelas da sociedade brasileira e do governo a respeito das melhores estratégias para se pensar no uso do IDH como um instrumento confiável e justo para a melhoria das políticas públicas no país (PNUD, 2010).

A análise do IDH-M dos municípios que constituem a Região Administrativa de Ribeirão Preto, também foi feita com a desagregação do índice, proporcionando uma verificação separada das variáveis (educação, longevidade e renda). Isso facilita a observação do desempenho dos indicadores de maneira mais detalhada (ANAND; SEN, 1994).

O objetivo da pesquisa foi analisar o nível de desenvolvimento humano dos municípios que compõem a RARP por meio do IDH-M, utilizando dados secundários, evidenciando os municípios com os indicadores mais críticos que compõem o índice, e apontar as principais potencialidades e fragilidades no uso desta ferramenta.

\section{INDICE DE DESENVOLVIMENTO HUMANO (IDH)}

O conceito de desenvolvimento humano pode ser entendido como um processo de ampliação das escolhas das pessoas, para que elas tenham capacidades e oportunidades para serem aquilo que desejam ser (SEN, 1993; PNUD, 2013a, 2013b). Amartya Sen (2000) defende que o desenvolvimento tem de estar relacionado, sobretudo, com a melhoria da vida e das liberdades. O conceito de liberdade está diretamente ligado às liberdades substantivas e instrumentais acessíveis à população de um dado território. As liberdades substantivas são entendidas por condições elementares de uma vida digna, como não ser acometido por: uma morte prematura, morbidez evitável, fome crônica ou ser tolhido de participação política, falta de liberdade de expressão ou ter negado o direito a condições mínimas de desenvolver a leitura e a escrita. Já, as liberdades instrumentais estão relacionadas às liberdades políticas, facilidades econômicas, oportunidades sociais, garantias de transparência e segurança (ORSI; GODOY, 2010).

Valiakhmetov (2015) se refere ao desenvolvimento humano como a expansão da liberdade das pessoas para desfrutar de uma vida longa, saudável e criativa, e que, individualmente ou em grupos, a participação do ser humano é essencial para assegurar a sustentabilidade do planeta.

Santos (2007) defende que o conceito de desenvolvimento humano é profundamente mais rico que qualquer índice ou conjunto de indicadores possa captar, dando essa afirmação como verdade também para outros indicadores. Para ele, alguns índices são úteis, ao focalizarem a atenção e simplificarem o problema, além de serem mais poderosos para chamar a atenção pública do que uma longa lista de indicadores combinada com uma discussão qualitativa.

O Índice de Desenvolvimento Humano (IDH) é a média aritmética de indicadores de três dimensões do desenvolvimento (educação, longevidade e renda). Por considerar que esse é o tripé sine qua non para a obtenção de todos os demais aspectos da qualidade de vida, e que são inseparáveis à proposta de crescimento, podemos perceber que não é linear a relação entre crescimento econômico e desenvolvimento, sendo importante dar o mínimo de atenção as suas discrepâncias (VEIGA, 2007).

O IDH foi destaque internacional pela sua capacidade de síntese e comunicação de aspectos importantes do desenvolvimento, enfatizando problemas considerados crônicos (distribuição de renda, gênero, pobreza, dentre outros). 
Desde a sua criação, o IDH, não tem sido poupado de críticas (KELLEY, 1991; SAGAR; NAJAM, 1998; IVANOVA; ARCELUS; SRINIVASAN, 1999; CHAKRAVARTY, 2011; RAVALLION, 2011; SRINIVASAN, 2012), já que até mesmo um de seus formuladores, Amartya Sen (prêmio Nobel de Economia de 1998), demonstrou-se bastante resistente quanto ao poder do índice de captar a realidade sobre o desenvolvimento humano (SANTOS, 2007). Veiga (2008) dispara algumas críticas ao índice, questionando os aspectos inerentes da composição do IDH, apontando como principal defeito metodológico o uso de média aritmética de três índices específicos (renda, escolaridade e longevidade), e a ausência de outras dimensões do desenvolvimento, como a ambiental, a cívica e a cultural. Jopp (2017) defende a substituição da média aritmética pela média geométrica com o objetivo de resolver o problema da substituibilidade perfeita, e também considera que o IDH negligencia outras dimensões importantes, como o da liberdade econômica, por exemplo.

Morse e Vogiatzakis (2014) consideram que o IDH não transparece o desenvolvimento humano (ou privação), pois possui poucos componentes na sua estrutura. Para os mesmos autores, a decisão de incluir poucos componentes no índice foi propositalmente feita pelo PNUD, com a intenção de tornar a métrica simples e transparente. McGillivray e White (1993) defendem que o índice é útil para comparar grupos de países com características semelhantes, mas, em termos de comparação entre países muito diferentes, apresenta deficiências. Em Kovacevic (2011) também se encontra uma visão crítica em relação ao uso do IDH, principalmente na consideração das variáveis empregadas.

Esforços para inclusão de mais variáveis no cálculo do IDH, como por exemplo da dimensão ambiental, têm sido realizados. Um exemplo é o Índice de Desenvolvimento Humano Sustentável (IDHS), que tem como proposta alterar o IDH, mas que é amplamente ignorado pela comunidade científica (BRAVO, 2014). A inclusão de dimensões e variáveis ambientais englobaria as recentes preocupações da sociedade contemporânea e contribuiria para a melhoria do índice (ALKIRE, 2010). Em relação à dimensão ambiental, Neumayer (2001, 2010) propõe acrescentar uma dimensão de sustentabilidade usando uma medida de investimento líquido em capital físico e natural, não subestimando o potencial de a sociedade garantir para as gerações futuras altos níveis de desenvolvimento humano.

As dimensões atribuídas no índice podem ser analisadas em níveis mais detalhados. A desagregação é importante para revelar as disparidades dentro do índice (ANAND; SEN, 1994), principalmente para a proposta do IDH em nível municipal. Essa desagregação dos índices sintéticos é importante para visualizar e analisar as variáveis críticas (observando as temáticas), que espelham as realidades associadas ao tema, para que se permita a comunicação com os usuários-agentes do poder público e privado (VEIGA, 2008).

Apesar das limitações do IDH, é oportuno ressaltar que esse indicador tem servido de estímulo ao surgimento de indicadores mais aperfeiçoados, em que, a partir do IDH, outros indicadores foram desenvolvidos (IDG - índice de desenvolvimento ajustado ao gênero; o MPG - medida de participação segundo o gênero; o IPH - índice de pobreza humana; o IDF - índice de desenvolvimento da família; o IPRS - índice paulista de responsabilidade social; e o IDH-M índice de desenvolvimento humano municipal), com o propósito de se aproximar da realidade dos indivíduos, não perdendo de vista o objetivo principal: captar o progresso humano por meio de dimensões básicas, como longevidade, educação e renda (SANTOS, 2007).

É importante ressaltar, na metodologia do IDH, que as comparações entre indicadores, municípios e anos (dados de 1991, 2000 e 2010) devem ser feitas somente a partir da plataforma 
Atlas Brasil. Nessa plataforma, o IDH-M é calculado com a metodologia recente, que possui as atualizações e ajustes necessários dos indicadores componentes, o que possibilita uma análise das tendências do desenvolvimento humano nos municípios. Também se faz importante salientar que é errado fazer qualquer tipo de comparação entre o IDH-M de um município e o IDH de um país, pois existem diferenças metodológicas em funções de ajustes sofridos para melhor adequação à realidade brasileira (PNUD, 2013b).

\section{INDICE DE DESENVOLVIMENTO HUMANO MUNICIPAL (IDH-M)}

O IDH é uma métrica que serve para comparações internacionais e auxilia o desenvolvimento de políticas públicas. Dada sua importância e utilidade, foi proposto um índice que seja medido e usado em níveis mais detalhados: o Índice de Desenvolvimento Humano Municipal. No Brasil, IDH-M (IDH com dados municipais) foi desenvolvido em 2003, com base em informações coletadas no Censo de 2000, sintetizado no formato do Atlas do Desenvolvimento Humano Municipal, com muitas edições específicas feitas para grandes metrópoles brasileiras. A partir daí, criou-se uma nova demanda além dos dados tradicionais, pois esses, ao serem dependentes do Censo, limitaram sua atualização a períodos de dez anos. Essa nova demanda de dados do IDH-M surgiu da necessidade de se poder contar com dados do IDH que sirvam para o monitoramento de políticas públicas locais e regionais, que contemplem horizontes de prazo mais curto, mais relacionados à configuração de políticas econômicas e sociais (PNUD, 2010).

$\mathrm{O}$ IDH-M oferece um resultado mais abrangente do desenvolvimento humano e tem impacto nos processos participativos e de gestão de políticas públicas. Também pode ser considerada uma importante ferramenta de referência para o planejamento municipal. A importância desse índice pode ser exemplificada quando os tomadores de decisão decidem usá-lo para direcionar ações públicas em diferentes áreas (PNUD, 2000; SANTA'ANNA; RIBEIRO; DUTT-ROSS, 2011; RAHAYU; RAVIYANTI; MAHARDIKA, 2016), e para tornar os municípios lugares cada vez melhores para se viver. $O$ índice em nível municipal também se mostra como um apoio importante para o entendimento das possíveis problemáticas em cada dimensão sintetizada em escala local (AGOSTINI; RICHARDSON, 1997).

A utilização de índices em escalas geográficas menores contribui de maneira mais efetiva para a promoção e o desenvolvimento humano local. Isso leva a uma avaliação mais detalhada dos níveis de desenvolvimento, auxiliando na definição de áreas prioritárias para recebimento de recursos (PAGLIANI, 2010).

Para Veiga (2008), as vantagens da municipalização do Índice de Desenvolvimento Humano (IDH-M) se perdem se os usuários não forem advertidos sobre os cuidados indispensáveis à sua utilização, para que não se tirem conclusões precipitadas sobre o desempenho dos municípios, a partir de simplórias comparações, atentando-se para o perigo que ele intitula "ranking-mania" (VEIGA, 2008). Esse cuidado com o índice é pertinente pelo fato de ser composto por indicadores, em que se deve levar em consideração que "os indicadores são de fato um modelo da realidade, mas não podem ser considerados a própria realidade, entretanto devem ser analiticamente legítimos e construídos dentro de uma metodologia coerente de mensuração" (BELLEN, 2006, p.45).

Ao refletir sobre o desenvolvimento em sua multiplicidade, tentando afastar visões reducionistas que são polarizadas unicamente nos aspectos socioeconômicos, ou nos aspectos físico-naturais, parte-se da hipótese de que o IDH e o IDH-M, embora apontem uma série de 
deficiências socioeconômicas importantes, refletem um resultado incoerente com um conceito de desenvolvimento abrangente, o qual se acredita que deva contemplar as dimensões sociais, econômicas e físico-naturais de maneira indissociável e seja voltado para a qualidade de vida das pessoas (ORSI; GODOY, 2010). Considerando o formato conceitual atual do IDH-M, os governos regionais e locais devem intensificar suas ações para uma distribuição equitativa do desenvolvimento humano nos municípios, regiões e até mesmo no país (RAHAYU; RAVIYANTI; MAHARDIKA, 2016).

\section{MATERIAIS E MÉTODOS}

A pesquisa desenvolvida tem característica exploratória, a partir de literatura especializada, relatórios, coleta e análise de dados secundários disponibilizados pelo website do Programa das Nações Unidas para o Desenvolvimento (PNUD), permitindo a triangulação dos dados para compor a análise. Também é descritiva, pois apresenta, sistematicamente, fatos e características presentes em uma determinada população ou área de interesse, não sendo simplesmente uma tabulação de dados, de modo que requer um elemento interpretativo que se apresenta combinando, muitas vezes, comparação, contraste, mensuração, classificação, interpretação e avaliação. O objeto de estudo, a Região Administrativa de Ribeirão Preto (RARP), foi escolhido por ser historicamente uma região de elevados índices de desenvolvimento em relação a outras regiões do Brasil, com cidades de produtividade importante no setor do agronegócio nacional e internacional, principalmente na produção de açúcar e álcool.

A RARP está localizada no centro-norte do Estado de São Paulo, Brasil. Ela é composta por 25 municípios, que ocupam uma área total de 934.800 ha, um total de 3,88\% do território paulista, e população em torno de 1.246 .000 habitantes (3,02\% da população paulista). Seu clima é tropical, relevo suave e plano, com predominância do solo chamado "terra roxa", que é resultante de alterações de rochas vulcânicas (formação Serra Geral) e arenito (formação Botucatu), considerados adequados ao desenvolvimento de agricultura. O agronegócio sucroalcooleiro é o principal indutor de desenvolvimento da economia nessa região, de segmentos industriais a ele relacionado, como o setor metal-mecânico ou o de turismo de negócios. A região é responsável por 3\% das exportações do Estado, possui um PIB de 47 bilhões de reais, que correspondem a 2,5\% do PIB paulista, e apresenta um PIB per capita de R\$35.754, 25 (SÃO PAULO, 2016).

A Figura 1 ilustra a delimitação espacial da RARP. 


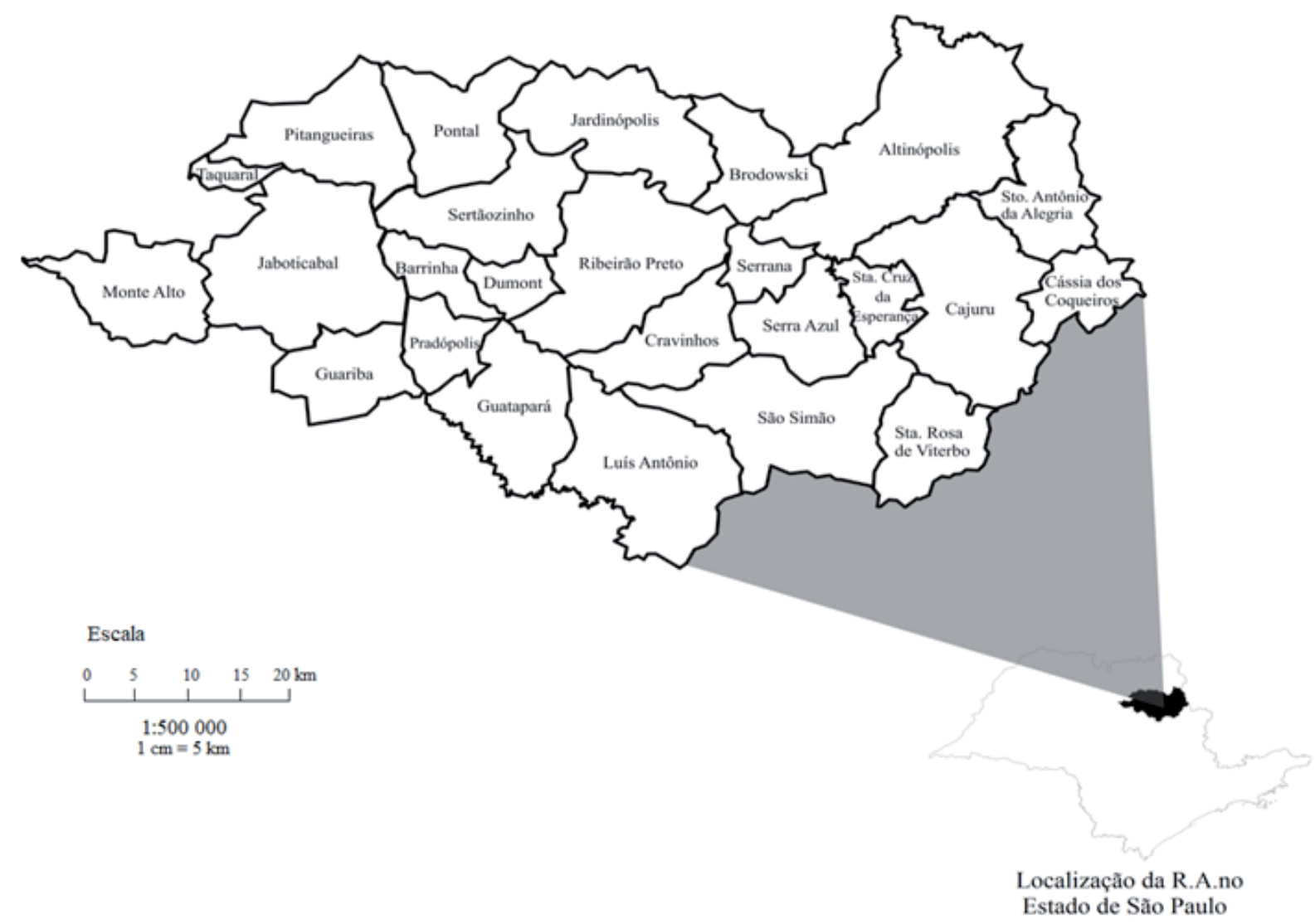

Figura 1 - Recorte espacial da pesquisa- Região Administrativa de Ribeirão Preto, SP Fonte: Adaptado pelos autores a partir de Instituto Geográfico e Cartográfico de São Paulo (IGC, 2007).

\section{RESULTADOS E DISCUSSÃO}

No âmbito municipal, observando a Figura 2, podemos visualizar os retratos situacionais do escopo escolhido para a análise, mostrando a distribuição das faixas de desenvolvimento humano, de acordo com dados do PNUD (2013a). 


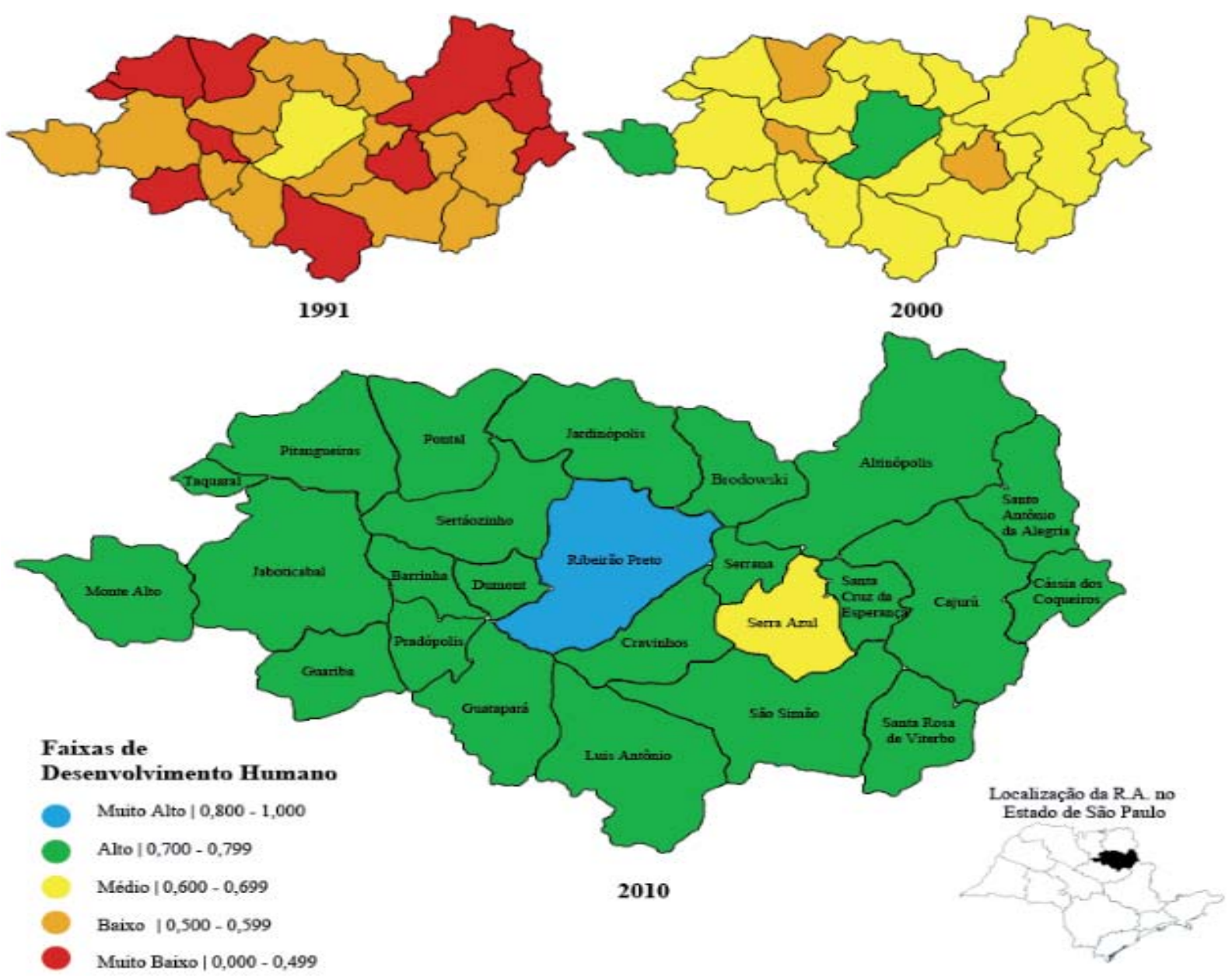

Figura 2 - Evolução do Índice de Desenvolvimento Humano Municipal na Região Administrativa de Ribeirão Preto no período de 1991-2010

Fonte: Elaborado pelos autores com dados de PNUD (2013a).

A partir de dados do PNUD (2013a), em 1991, 96\% da Região Administrativa de Ribeirão Preto estavam na faixa de classificação de desenvolvimento humano entre "muito baixo" e "baixo", com apenas um dos municípios fora dessa zona (Ribeirão Preto, com IDH-M de 0,626). Nesse período, o município que teve pior desempenho na RARP foi Barrinha, com IDH-M de 0,409. No ano de 2000, a cidade de Barrinha repetiu o pior resultado da RARP, atingindo o valor de 0,591, mesmo apresentando uma evolução de 18,2\% entre os períodos de 1991-2000. Ribeirão Preto obteve novamente o melhor desempenho, com o valor 0,733 (aumento de 10,7\% em relação a 1991). Em 2010, Ribeirão Preto continuou com o melhor desempenho da região $(0,800)$, e a cidade de Serra Azul foi a localidade que ficou na pior colocação, com o IDH-M de 0,686. No período de 1991-2000, todos os municípios da RARP elevaram seus índices de desenvolvimento humano, e a média desse aumento do IDH-M foi de 13,12\% para a região. O município que se destacou com o melhor desempenho, no período de 1991-2000, foi Luís Antônio (de 0,481 para 0,672), com uma evolução de 19,1\%, e Pradópolis ficou com o desempenho mais baixo no mesmo período (8,6\%). No período de 2000-2010, a cidade de Barrinha obteve a evolução mais considerável, melhorando seu IDH-M em 13,4\% (saltou de 0,591 para 0,725 pontos), e o município de Luís Antônio apresentou o valor mais modesto, de 5,9\% (de 0,672 para 0,731 pontos). No valor acumulado de 1991-2010, a cidade de Barrinha protagonizou a melhor pontuação do 
período, com uma evolução de 31,6\% (0,409 para 0,725), e Ribeirão Preto portou a crescente de $17,4 \%$, considerada no período acumulado o menor valor.

Na Tabela 1, estão elencados ordinalmente os valores do IDH-M de 2010, de cada cidade que compõe a RARP, e sua respectiva classificação, considerando todos os municípios brasileiros de acordo com dados do PNUD (2013a).

Tabela 1 - Valores do IDH-M das cidades da RARP e a posição em relação aos municípios brasileiros

\begin{tabular}{|c|c|c|}
\hline & IDH-M (2010) & Classificação no Brasil (2010) \\
\hline Ribeirão Preto & 0,800 & 409 \\
\hline Jaboticabal & 0,778 & 1450 \\
\hline Santa Rosa de Viterbo & 0,770 & 2270 \\
\hline Monte Alto & 0,768 & 249o \\
\hline São Simão & 0,766 & 2749 \\
\hline Sertãozinho & 0,761 & 3500 \\
\hline Taquaral & 0,759 & 383응 \\
\hline Cravinhos & 0,756 & $440=$ \\
\hline Brodowski & 0,755 & 453응 \\
\hline Dumont & 0,744 & 6670 \\
\hline Guatapará & 0,743 & 6950 \\
\hline Santa Cruz da Esperança & 0,743 & 6950 \\
\hline Jardinópolis & 0,735 & 8970 \\
\hline Cássia dos Coqueiros & 0,734 & 9200 \\
\hline Pradópolis & 0,733 & 940 - \\
\hline Luís Antônio & 0,731 & 993음 \\
\hline Altinópolis & 0,730 & 10219 \\
\hline Serrana & 0,729 & 10529 \\
\hline Pontal & 0,725 & 11549 \\
\hline Barrinha & 0,725 & 11540 \\
\hline Pitangueiras & 0,723 & 12170 \\
\hline Guariba & 0,719 & 13310 \\
\hline Cajuru & 0,713 & 15140 \\
\hline Santo Antônio da Alegria & 0,702 & $1842 \circ$ \\
\hline Serra Azul & 0,686 & $2282 \circ$ \\
\hline
\end{tabular}

Fonte: Elaborado pelos autores a partir de dados do PNUD (2011; 2013a).

Dentre os municípios do Estado de São Paulo, a cidade de Ribeirão Preto, a melhor colocada na RARP em 2010, ficou com o IDH-M inferior aos valores alcançados pelos municípios de São Caetano do Sul $(0,862)$, Águas de São Pedro $(0,854)$, Santos $(0,840)$, Jundiaí $(0,822)$, Valinhos $(0,819)$, Vinhedo $(0,817)$, Santo André $(0,815)$, Araraquara $(0,815)$, Santana de Parnaíba $(0,814)$, Ilha Solteira $(0,812)$, Americana $(0,811)$, São José dos Campos $(0,807)$, Presidente Prudente $(0,806)$, São Paulo $(0,805)$, Assis $(0,805)$, Campinas $(0,805)$, São Bernardo do Campo $(0,805)$, São Carlos (0,805), Rio Claro (0,803), Bauru $(0,801)$ e Pirassununga $(0,801)$. A cidade de Taubaté possui o mesmo IDH-M de Ribeirão Preto, mas leva vantagem no tema Longevidade $(0,883)$ e no tema Educação $(0,746)$.

Dentre as dez primeiras posições ocupadas no Ranking IDH-M dos municípios brasileiros em 2010, três são de municípios paulistas. Destaque para o município de São Caetano, que encabeça a lista (PNUD, 2013a), seguido por Águas de São Pedro (2a posição) e Santos (6a posição). 
Em 2010, a RARP apresentou uma tendência de homogeneidade nos resultados do IDH-M, o que pode caracterizar uma sincronia das ações dos tomadores de decisão e atores sociais em torno do desenvolvimento humano. Isso aponta que os pontos de inflexão observados em períodos anteriores foram articulados de forma satisfatória e afloraram perspectivas positivas para propiciar à sociedade bons níveis de bem-estar. Ações políticas, que fomentem a participação de todos os interessados, desenvolvidas localmente, podem auxiliar a construção de estruturas culturais, legais e políticas, que ampliam o exercício da cidadania, introduzindo valores que tem alcance regional. Isso abre espaço para se pensar em planejamentos urbanos ou setoriais de forma mais abrangente e coerente. É fato que os planejamentos, planos diretores, aparatos legais e outras ferramentas de gestão, pensados localmente, têm influência em localidades vizinhas e devem ser pensados de forma mais complexa e interativa.

Ao se fazer a decomposição do IDH-M, conseguimos perceber as variáveis críticas que compõem o índice, resultando na melhor análise dos temas considerados, oferecendo assim, melhores condições de compreensão aos usuários e interessados. No Gráfico 1, estão contidos dados de cada variável sintetizado no IDH-M de 2010, evidenciando o desempenho de cada uma delas isoladamente.

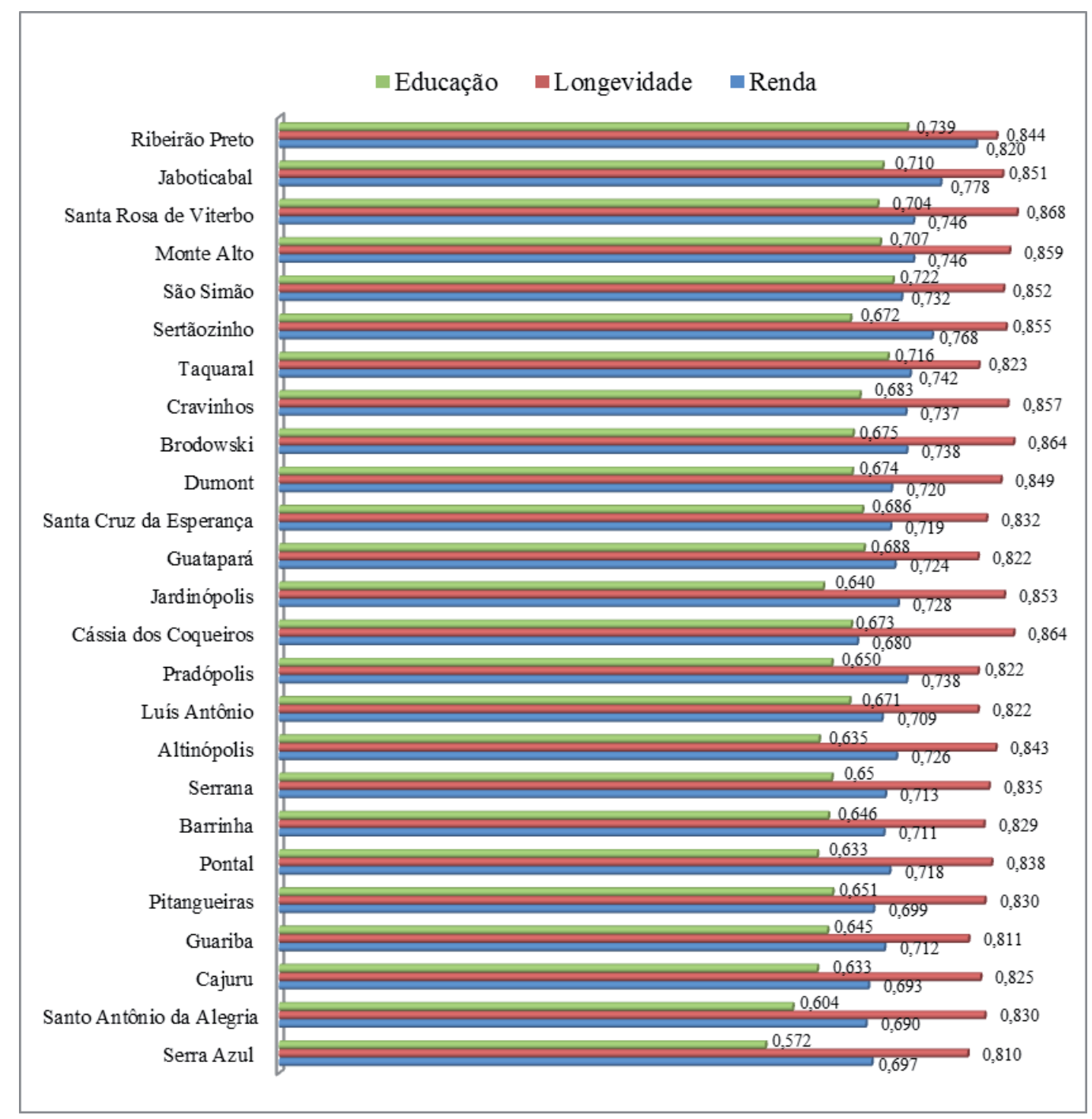

Gráfico 1 - Apresentação e desempenho das variáveis que compõem o IDH-M no ano de 2010 na RARP

Fonte:Elaborado pelos autores a partir de dados do PNUD (2013a). 
Analisando os valores das variáveis do tema Educação da RARP, expostas no Gráfico 1, a localidade que apresentou o pior desempenho foi o município de Serra Azul. De acordo com o PNUD (2013a), Serra Azul ocupa a 2282a posição, em 2010, em relação aos 5.565 municípios do Brasil, sendo que 2281 municípios (40,99\%) estão em situação melhor e 3.283 municípios (58,99\%) estão em situação igual ou pior. Em relação aos 645 outros municípios do Estado de São Paulo, a mesma cidade ocupa a 614a posição, sendo que 613 municípios (95,04\%) estão em situação melhor e 31 municípios (4,81\%) estão em situação pior ou igual (PNUD, 2013a). O tema Educação, para a cidade de Serra Azul, está classificado como baixo (valor entre 0,500 - 0,599), sendo o único dentre os municípios participes da RARP a estar nessa faixa de desenvolvimento. Dentre os cinco indicadores que compõem o tema Educação, o mais crítico para a cidade de Serra Azul foi o indicador Percentual de alunos de 18 a 20 anos com ensino médio completo, com o resultado de 29,93\%. De acordo com o PNUD (2013a), a proporção para esse indicador cresceu $12,86 \%$ entre 2000 e 2010, e 180,63\% entre 1991 e 2000. O indicador com melhor desempenho foi o que aponta o percentual de alunos de 5 a 6 que frequentam a escola, com 93,46\%, seguido pelos indicadores percentuais de alunos de 11 a 13 anos frequentando os anos finais do ensino fundamental (74,67\%), de alunos de 15 a 17 anos com ensino fundamental completo (64,36\%) ede alunos com 18 anos ou mais com ensino fundamental completo (43,62\%) (PNUD, 2013a). De acordo com o relatório de gestão da Secretaria da Educação do município, em 2010, 28,32\% da receita resultante dos impostos foi direcionada à manutenção e desenvolvimento do ensino público (SERRA AZUL, 2014), em conformidade com o artigo 212 da Constituição Federal (BRASIL, 2014). O que se observa é que o valor temático alcançado para a Educação, para o município de Serra Azul, apontou resultado similar ao encontrado em municípios da costa oeste do Paraná,que apresentaram baixos valores no mesmo tema e no índice geral (CASAGRANDE; SOUZA, 2013). No sudoeste europeu, a região de Portugal chamada NUTS III (nomenclatura das unidades territoriais para fins estatísticos), apresentou dispersão nos outros resultados do desenvolvimento humano, menos no tema Educação, o que demonstra uma forte política regional acerca desse tema (SILVA; FERREIRA-LOPEZ, 2014).

Para atingir níveis satisfatórios de desenvolvimento no tema Educação, é necessário otimizar as atividades voltadas para essa dimensão de forma ampla. Variáveis múltiplas necessitam ser objeto de preocupação do poder público nesse sentido, e incorporadas na agenda políticas em todos os níveis (BATALHÃO; TEIXEIRA, 2013).

No tema Longevidade, que é composto pelo indicador Esperança de vida ao nascer (em anos), o município de Serra Azul aparece novamente com o pior resultado em 2010. O indicador Esperança de vida ao nascer aumentou 7,4 anos nas últimas duas décadas, passando de 66,2 anos em 1991 para 69,2 anos em 2000, e para 73,6 anos em 2010 (PNUD, 2013a). Esse incremento, que representa um valor baixo perante as outras cidades da RARP, poderia ser maior se as outras variáveis que incidem interferência no indicador de Longevidade tivessem melhor desempenho. Valiakhmetov (2015) argumenta que o tempo de vida da população, e a proporção de pessoas ativas economicamente (pensando no contexto previdenciário) influenciam o padrão de desenvolvimento. Isso pode resultar numa dificuldade do Estado em prover a satisfação das necessidades da população (saúde, cultura, educação) e assegurar seus direitos de forma justa e assertiva.

Para atingir valores aceitáveis e sustentáveis no tema Longevidade, é necessário observar um conjunto de características constitutivas e a disponibilidade de serviços básicos de infraes- 
trutura, saúde, saneamento, dentre outros. Esses fatores são determinantes para o bem-estar social de uma localidade ou região. A camada socioeconômica em que o indivíduo está inserido, o nível de instrução e a disposição de equipamentos de saúde (geograficamente) podem contribuir para a evolução do tema Longevidade. O acompanhamento da atenção básica nas localidades também exerce impacto positivo na saúde das pessoas, e consequentemente, na continuidade das atividades produtivas.

O tema Renda é composto pelo indicador Renda per capita (em R\$). O município de Cássia dos Coqueiros apresentou o menor valor na RARP no tema renda, com o valor de 0,680, mesmo tendo o seu IDH-M classificado na faixa de desenvolvimento Alto $(0,734)$ em 2010 . Segundo o PNUD (2013a), a renda per capita média de Cássia dos Coqueiros cresceu 62,93\% nas últimas duas décadas, passando de R\$338,42 em 1991 para R\$ 463,70 em 2000 e R\$ 551,40 em 2010. Esse indicador auxilia no entendimento de padrões de consumo e produção, considerado um dos indicadores básicos para observar o comportamento de uma economia. O baixo desempenho deste indicador na cidade de Cássia dos Coqueiros evidencia que a renda média dessa população em 2010, se mostrou insuficiente, podendo ter como fator impactante a desigualdade em sua distribuição em escala local.

A articulação das três dimensões que compõem o IDH-M realçaram de forma negativa o desempenho do município de Serra Azul. Mesmo não apresentando no tema Renda o menor valor, o IDH-M geral é o pior da região.

Para os resultados da análise feita na RARP com o IDH-M, sugerimos algumas hipóteses com teor explicativo com o objetivo de justificar as disparidades intrarregionais encontradas na pesquisa, e propor possíveis ações corretivas nesse âmbito. Aqui, as disparidades são entendidas como desigualdades no nível das condições de vida das famílias inseridas nos municípios, que podem retardar o desenvolvimento regional. No Quadro 1, estão dispostas as hipóteses, as principais disparidades e as propostas de ações corretivas para a RARP. 


\begin{tabular}{|c|c|c|}
\hline Hipóteses & Disparidades & Ações corretivas \\
\hline $\begin{array}{l}\text { A base institucional na } \\
\text { região está enfraquecida }\end{array}$ & $\begin{array}{c}\text { Baixo grau de articulação política } \\
\text { entre os municípios; } \\
\text { Falta de mecanismos de } \\
\text { articulação institucional; } \\
\text { Incongruência administrativa }\end{array}$ & $\begin{array}{l}\text { Legitimação dos interesses e } \\
\text { demandas da região por meio de } \\
\text { instrumentos legais }\end{array}$ \\
\hline $\begin{array}{l}\text { A região é dependente da } \\
\text { monocultura canavieira }\end{array}$ & $\begin{array}{c}\text { Desemprego sazonal e } \\
\text { incapacidade produtiva imposta } \\
\text { ao trabalhador rural (gerando } \\
\text { excedente populacional); } \\
\text { Substituição da mão-de-obra } \\
\text { braçal por mecanizada; } \\
\text { Desigualdades sociais, diminuindo } \\
\text { a capacidade financeira de } \\
\text { emancipar realizações }\end{array}$ & $\begin{array}{l}\text { Incentivar a heterogeneidade } \\
\text { econômica intra-regional } \\
\text { (diversificação de produção) } \\
\text { e fomentar mecanismos de } \\
\text { distribuição de investimentos } \\
\text { pelos poderes públicos e privados }\end{array}$ \\
\hline $\begin{array}{l}\text { As políticas públicas } \\
\text { regionais estão } \\
\text { enfraquecidas }\end{array}$ & $\begin{array}{c}\text { Desequilíbrio econômico nos } \\
\text { municípios periféricos (falta de } \\
\text { incentivos fiscais, tecnologia, infra- } \\
\text { estrutura) }\end{array}$ & $\begin{array}{c}\text { Dinamizar a economia dos } \\
\text { municípios periféricos e socializar } \\
\text { tecnologias de produção, ciência } \\
\text { e logística }\end{array}$ \\
\hline $\begin{array}{l}\text { Existe uma } \\
\text { incomunicabilidade de } \\
\text { alguns municípios com } \\
\text { as metas nacionais da } \\
\text { educação }\end{array}$ & $\begin{array}{l}\text { Baixo rendimento escolar em } \\
\text { alguns municípios da região, não } \\
\text { atendendo as metas estratégicas } \\
\text { estabelecidas pelo Ministério da } \\
\text { Educação para cada município }\end{array}$ & $\begin{array}{l}\text { Criação de uma métrica pra } \\
\text { acompanhar o desenvolvimento } \\
\text { da educação intra-regional }\end{array}$ \\
\hline $\begin{array}{l}\text { Projetos públicos e } \\
\text { privados para a integração } \\
\text { regional são engavetados }\end{array}$ & $\begin{array}{l}\text { Ausência de investimentos em } \\
\text { municípios distantes do centro; } \\
\text { Pouca efetividade na criação de } \\
\text { câmaras técnicas e conselhos } \\
\text { participativos para tratar da } \\
\text { integração regional; } \\
\text { Pouca aderência dos líderes } \\
\text { políticos na priorização do tema } \\
\text { na agenda de governo local }\end{array}$ & $\begin{array}{c}\text { Promover uma abordagem } \\
\text { intervencionista por parte de } \\
\text { instituições funcionais públicas } \\
\text { para legitimar os interesses } \\
\text { regionais }\end{array}$ \\
\hline $\begin{array}{c}\text { A centralização das } \\
\text { atividades produtivas } \\
\text { polariza o desenvolvimento } \\
\text { e não dinamiza a região de } \\
\text { maneira igualitária }\end{array}$ & $\begin{array}{l}\text { Incapacidade de geração de } \\
\text { renda por parte dos municípios } \\
\text { periféricos, e conseqüentemente, } \\
\text { baixa arrecadação tributária e } \\
\text { baixo nível de investimento em } \\
\text { equipamentos públicos por estes }\end{array}$ & $\begin{array}{l}\text { Reversão da polarização intra- } \\
\text { regional por meio de um } \\
\text { processo de desconcentração e } \\
\text { descentralização das atividades } \\
\text { econômicas dinâmicas para } \\
\text { todos os municípios da região } \\
\text { considerando suas subjetividades } \\
\text { (harmonização e sinergia) }\end{array}$ \\
\hline
\end{tabular}

Quadro 1 - Hipóteses, principais disparidades e propostas de ações corretivas para a RARP a partir do IDH-M

Fonte: Elaborado pelos autores.

As hipóteses apontadas acima correspondem aos desafios encontrados na análise feita, e demonstram a urgência de engendrar ações mais ágeis e sistemáticas para promover a monitorização das condiç̧̃es municipais.

Avaliações e reflexões sobre o espaço municipal desempenham um papel fundamental na elaboração de planos de desenvolvimento que incluam simultaneamente metas nacionais e re- 
gionais. Esses critérios são essencialmente importantes para conciliar a abordagem funcional, em termos de metas e objetivos nacionais globais, com a abordagem regional. Isso permite superar os problemas intrarregionais e mobilizar suas potencialidades de maneira reflexiva, utilizando a capacidade endógena de organização social, econômica e política para modelar cenários futuros.

No caso da RARP, a crescente centralização da tomada de decisões econômicas e políticas revelaram a interdependência entre a distribuição geográfica do bem-estar social e a estrutura espacial das atividades políticas e econômicas. Talvez a relação institucional entre os municípios da mesma região pode ter sido negligenciada em algum momento, desconsiderando a multiplicidade do desenvolvimento. Percebeu-se que é necessário investir tempo na agenda política e em arcabouços normativos para ampliar as alianças entre as administrações municipais, para que se tornem exequíveis ações corretivas e preventivas de abrangência regional, que exigem soluções em parceria. Isso significa que os municípios precisam se unir para resolver problemas e dimensionar demandas de interesse comum. Um novo arranjo institucional deve ser o instrumento de um planejamento regional eficiente, promovendo cooperações, evitando desperdícios de recursos e reduzindo as grandes desigualdades intrarregionais. Um planejamento conjunto integrado a uma nova política regional pode ajudar a diminuir as assimetrias nas dimensões do desenvolvimento entre os municípios e compatibilizar os interesses coletivos, garantindo uma forma mais justa de democratização dos benefícios econômicos e sociais.

Essa nova política regional deve ser guiada pela integração territorial, econômica, social e política. A representação política necessita preservar a relação entre o recorte territorial e a identidade cultural e política dos munícipes e de suas instituições e, concomitantemente, os aspectos de geopolítica e soberania nacional (DINIZ, 2009). Entretanto cabe considerar que algumas regiões podem gerar reações negativas, tais como deslocamento populacional e degradação do meio-ambiente, e não criar efeitos positivos que anulem essas reações adversas (LEMOS et al., 2005).

Conforme afirma Furtado (1967), na prática, as tentativas de conciliar os vários critérios derivados de análises setoriais ou regionais continuam sendo principalmente uma questão de decisão política. No entanto a possibilidade de dar a essas decisões uma base mais racional aumenta à medida que a análise econômica se une a outras dimensões do desenvolvimento para promover o entendimento das estruturas espaciais. O mesmo autor também defende que é necessário superar o padrão espacial de "região", promovendo-o para uma estrutura espacial com articulações intraterritorial e interterritorial, com ligações funcionais organizadas por uma hierarquia que envolva a complexidade e intensidade das relações entre agentes e atores no território.

A partir da experiência com a RARP e considerando outras experiências observadas e analisadas na revisão de literatura desenvolvida para este trabalho, no Quadro 2 foram distribuídas as fragilidades e potencialidades do IDH-M, com o propósito de dar continuidade a reflexões em torno dessa ferramenta e traçar um panorama crítico com aspectos encontrados em literatura. 


\begin{tabular}{|c|l|}
\hline \multirow{5}{*}{ Fragilidades } & Não representa todas as demandas dos municípios \\
\cline { 2 - 3 } & $\begin{array}{l}\text { Contempla poucas variáveis, o que limita a análise da complexidade do } \\
\text { desenvolvimento humano }\end{array}$ \\
\cline { 2 - 3 } & Ausência de outras dimensões do desenvolvimento humano, como a ambiental \\
\cline { 2 - 3 } & Promove uma generalização de realidades municipais distintas \\
\cline { 2 - 3 } & Não considera especificidades culturais e geográficas dos municípios \\
\cline { 2 - 3 } & Apresenta correlação fraca com gestão de recursos e programas sociais \\
\hline \multirow{4}{*}{ Potencialidades } & Permite análise detalhada das dimensões do índice \\
\cline { 2 - 3 } & Promove monitoramento das políticas públicas municipais \\
\cline { 2 - 3 } & Não gera sobreposição ou distorção dos resultados unidimensionais e do índice \\
\cline { 2 - 3 } & Permite e promove efeito comparativo \\
\cline { 2 - 3 } & É de uso estratégico para o processo decisório \\
\cline { 2 - 3 } & Tem apresentado crescente nível de usuabilidade por governos \\
\cline { 2 - 3 } & Promove ações corretivas e de planejamento municipal \\
\hline
\end{tabular}

Quadro 2 - Fragilidades e Potencialidades encontradas em iniciativas com IDH-M Fonte: Elaborado pelos autores.

Existe, de fato, um paradoxo no pensamento complexo sobre a interação das dimensões do desenvolvimento humano e quais incrementos podem gerar impactos, positivos ou negativos, de maior ou menor espectro. A notoriedade de que as questões do desenvolvimento humano passaram a ser progressivamente incorporadas nos processos de transformação e evolução tecnológica e econômica também traz consigo a necessidade de (re) orientação de metas e objetivos de políticas públicas setoriais.

Os resultados corroboram com Veiga (2007), quando percebemos que as relações entre desenvolvimento e economia não são lineares. O mesmo autor argumenta que a expansão da educação básica e dos serviços de saúde não precisam vir depois do enriquecimento do Estado, isso acontece com o aumento das disposições sociais nas economias pobres. Também corroboram com Jopp (2017) e Veiga (2008) mostrando que é necessária uma revisão metodológica do IDH-M para ser uma ferramenta factível e de aplicabilidade flexível, e não esquecer outras dimensões tão importantes e indispensáveis como as já empregadas no índice.

\section{CONSIDERAÇÕES FINAIS}

Neste artigo, buscou-se pensar regionalmente, observando dados resultantes da utilização de instrumentos de gestão e de criação/manutenção de políticas públicas localmente. Cada variável que compõe o IDH-M representa o retrato situacional a ser considerado no processo de desenvolvimento de regiões, apesar de ser um instrumento de avaliação de desenvolvimento humano local.

As abordagens e análises desenvolvidas nesta pesquisa fizeram perceber que as características nos contextos culturais, sociais e geopolíticas são determinantes para os padrões de desenvolvimento humano. Os novos padrões tecnológicos, por exemplo, polarizados em determinadas regiões, causam impacto direto no nível econômico desses espaços geográficos. Percebe-se que a convergência das atuais demandas da sociedade contemporânea e o desenvolvimento humano precisam ser reavaliados e reestruturados, já que outros aspectos precisam ser considerados, como por exemplo,os aspectos ecossistêmicos. 
É importante lembrar que as informações sintetizadas pelo IDH-M apontam e servem de matéria-prima para um processo de transformação social, a fim de externar as necessidades da região e cravar na agenda política de cada município a importância da compatibilidade entre desenvolvimento humano e políticas públicas efetivas.

Para que se consiga o desenvolvimento regional, é necessário alcançar primeiro o desenvolvimento local. Os interesses nacionais, estaduais e locais devem estar sinergicamente interligados, para que os recursos sejam utilizados racionalmente, diminuindo as possíveis desigualdades. Mostra-se necessário um esforço coletivo, tanto na esfera pública quanto na privada, para que haja novas fontes de incentivo ao desenvolvimento local em ações empreendedoras, inclusivas e sustentáveis. Os municípios podem e devem se organizar para que consigam incrementar seus resultados no âmbito do desenvolvimento humano e consigam alavancar a qualidade de vida de sua população.

\section{REFERÊNCIAS}

AGOSTINI, S. J.; RICHARSON, S. J. A Human Development Index for U.S. cities: methodological issues and preliminary findings. Economics, v. 25, n. 1, p. 13-41, 1997.

ALKIRE, S. Human development: definitions, critiques, and related concepts. Human Development Research Paper. OPHI Working Paper, Oxford, n. 36, 2010.

ANAND, S .; SEN, A. Human Development Index: methodology and measurement. New York: Human Development Report Office (HDRO), United Nations Development Programme (UNDP), 1994.

BATALHÃO, A. C. S.; TEIXEIRA, D. Indicadores de Sustentabilidade para a Educação: uma análise do tema Conhecimento e Cultura a partir do Barômetro da Sustentabilidade em Ribeirão Preto (SP). In: ESPIRITOSANTO, P. S. M. F.; BANDOS, M. F. C. (Org.). Políticas públicas e desenvolvimento. Franca, SP: Editora UniFacef, 2013. p. 1-23.

BELLEN, H. M. Indicadores de sustentabilidade: uma análise comparativa. Rio de Janeiro: Editora FGV, 2006.

BRASIL. Constituição Federal 1988. Brasília: Senado Federal, 2014. Disponível em: <http://www.senado. gov.br/legislacao/const/con1988/con1988_13.07.2010/art_212_.shtm>. Acesso em: 6 jul. 2014.

PNDR I - Política Nacional de Desenvolvimento Regional. Brasília: Ministério da Integração Nacional, 2011. Disponível em: <http://www.mi.gov.br/politica-nacional-de-desenvolvimento-regionalpndr>. Acesso em: 20 set. 2017.

BRAVO, G. The Human Sustainable Development Index: news calculations and a first critical analysis. Ecological Indicators, v. 37, parte A, p. 145-50, fev. 2014.

BUARQUE, S. C. Construindo o desenvolvimento local sustentável. Rio de Janeiro: Garamond, 2008.

CASAGRANDE, A. E.; SOUZA, E. B. C. Do planejamento ao ordenamento territorial: estudo da região costa oeste do Paraná. Ra'e Ga, Curitiba, v. 28, p. 67-85, 2013.

CHAKRAVARTY, S. R. A reconsideration of the tradeoffs in the new Human Development Index. The Journal of Economic Inequality, v. 9, n. 3, p. 471-4, 2011.

DINIZ, C. C. Celso Furtado e o desenvolvimento regional. Revista Nova Economia, v. 19, n. 2, p. 227-49, 2009.

FURTADO, C. Desenvolvimento e subdesenvolvimento. Rio de Janeiro: Contraponto/Centro Internacional Celso Furtado, 2009.

. Raízes do subdesenvolvimento. Rio de Janeiro: Civilização Brasileira, 2003.

.Introdução ao desenvolvimento: enfoque histórico-estrutural. Rio de Janeiro: Paz e Terra, 2000.

.Cultura e desenvolvimento em época de crise. Rio de Janeiro: Paz e Terra, 1984.

INTERAÇÕES, Campo Grande, MS, v. 19, n. 2, p. 237-256, abr./jun. 2018 
. Intra-country discontinuities: towards a theory of spatial structures. Social Science Information, v. 6, n. 6, p. 7-14, 1967.

GOR, S. O.; GITAU, C. M. W. Rethinking the HDI: a more theoretically consistent alternative. International Journal of Sustainable Development, v. 1, n. 5, p. 85-90, 2010.

INSTITUTO GEOGRÁFICO E CARTOGRÁFICO DE SÃO PAULO (IGC). Mapas individuais das Regiões Administrativas e Metropolitanas. 2007. Disponível em: <http://www.igc.sp.gov.br/produtos/mapas_ ra.aspx?>. Acesso em: 20 set. 2017.

IVANOVA, I.; ARCELUS, F. J.; SRINIVASAN, G. An assessment of the measurement properties of the Human Development Index. Social Indicators Research, v. 46, n. 2, p. 157-79, 1999.

JOPP, T. A. Ein glücksökonomisch modifizierter Human Development Index für Deutschland (1920-1960). Jahrbuch fur Wirtschaftsgeschichte, v. 58, n. 1, p. 239-78, 2017.

KELLEY, A. C. The Human Development Index: "Handle with Care". Population and Development Review, v. 17, n. 2, p. 315-24, jun. 1991.

KOVACEVIC, M. Human Development Research Paper: review of HDI critiques and potential improvements. Nova lorque: UNDP, 2011.

LEMOS, M. B.; RUIZ, R. M.; MORO, S.; E DOMINGUES, E. P. Espaços preferenciais e aglomerações industriais. In: DE NEGRI, J. A.; SALERMO, M. (Ed.). Inovação, padrões tecnológicos e desempenho das firmas industriais brasileiras. Rio de Janeiro: Ipea, 2005.

MAZOUCH, P.; VLTAVSKÁ, K.; STAŇEK, T. Towards the unnecessity of Human Development Index: the case of sensitivity analysis. Statistika, v. 96, n. 4, p. 5-18, 2016.

MCGILLIVRAV, M.; WHITE, H. Measuring development? The UNDP's human development index. Journal of International Development, v. 5, n. 2, p. 183-92, 1993.

MORSE, S.; VOGIATZAKIS, I. N. Resource use and deprivation: geographical analysis of the ecological footprint and town send index for England. Sustainability, v. 6, n. 8. p. 4749-71, 2014.

NEUMAYER, E. Human development and sustainability. Human Development Research Paper. Londres: UNDP, 2010.

The human development index and sustainability - a constructive proposal. Ecological Economics, v. 39, p. 101-14, 2001.

ORSI, R. A.; GODOY, M. B. R. B. Reflexões sobre o desenvolvimento e a sustentabilidade: o que o IDH e o IDHM podem nos mostrar? In: FERREIRA, D. A. O.; FERREIRA, E. R. (Org.).Geografia e território: interpretações do espaço brasileiro. Rio Claro, SP: IGCE/UNESP - Pós-Graduação em Geografia, 2010. p. 217-35.

PAGLIANI, P. Influence of regional, national and sub-national HDRs. Human Development Research Paper 2010/19. United Nations Development Programme, 2010. Disponível em: <http://hdr.undp.org/en/reports/ global/hdr2010/papers/HDRP_2010_19.pdf>. Acesso em: 1o out. 2015.

PLANO DAS NAÇÕES UNIDAS PARA O DESENVOLVIMENTO (PNUD). PNUD analisa o impacto do IDHM na gestão pública brasileira. Brasília: PNUD, 2014a. Disponível em: <http://www.br.undp.org/content/brazil/ pt/home/presscenter/articles/2014/04/24/pnud-analisa-o-impacto-do-idhm-na-gest-o-p-blica-brasileira. html>. Acesso em: 8 jul. 2015.

Brasil sobe uma posição no ranking do IDH e fica em 79o entre 187 países. Brasília: PNUD, 2014b. Disponível em: <http://www.br.undp.org/content/brazil/pt/home/presscenter/articles/2014/07/24/ brasil-sobe-uma-posi-o-no-ranking-do-idh-e-fica-em-79-entre-187-pa-ses.html>. Acesso em: 26 jul. 2015.

. Atlas do Desenvolvimento Humano no Brasil 2013 - Consulta. Brasília: PNUD, 2013a. Disponível em: <http://atlasbrasil.org.br/2013/consulta/>. Acesso em: 24 mar. 2015.

. Desenvolvimento Humano e IDH. Brasília: PNUD, 2013b. Disponível em: <http://www.pnud.org. br/IDH/DesenvolvimentoHumano.aspx?indiceAccordion=0\&li=li_DH>. Acesso em: 23 dez. 2015. 
RDH 2011 - Sustentabilidade e equidade: um futuro melhor para todos. Nova lorque: PNUD, 2011. RDH 2009/2010 - valores e desenvolvimento humano. Brasília: PNUD, 2010.

.National Human Development Report Bulgaria 2000. The Municipal Mosaic. Human Development Report. UNDP, 2000. Disponível em: <http://hdr.undp.org/en/reports/national/europethecis/bulgaria/ bulgaria_2000_en.pdf>. Acesso em: 3 mar. 2015.

RAHAYU, S.; RAVIYANTI, A. A.; MAHARDIKA, D. P. K. Local government revenue, general allocation fund, special allocation fund, capital expenditure to Human Development Index (HDI) in Papua, Indonesia. Advanced Science Letters, v. 22, n. 12, p. 4077-81, 2016.

RAVALLION, M. The Human Development Index: a response to Klugman, Rodriguez and Choi. Journal of Economics Inequality, v. 9, n. 3, p. 475-8, 2011.

SAGAR, A. D.; NAJAM, A. The Human Development Index: a critical review. Ecological Economics, v. 25, n. 3, p. 249-64, 1998.

SANT'ANNA, A. P.; RIBEIRO, R. O. A.; DUTT-ROSS, S. Employing the components of the Human Development Index to drive resources to educational policies. Social Indicators Research, v. 104, n. 3, p. 523-32, 2011.

SANTOS, M. P. Distribuição de renda e desenvolvimento humano: estado de São Paulo 1970-2000. São Paulo: Educ, 2007.

SÃO PAULO, Estado [de]. Secretaria de Planejamento e Gestão. Desenvolvimento regional. São Paulo, 2016. Disponível em: <http://www.planejamento.sp.gov.br/index.php?id=50\&idd=163>. Acesso em: 12 mar. 2016.

SEN, A. Desenvolvimento como liberdade. São Paulo: Companhia das Letras, 2000.

Capability and well-being. In: NUSSBAUM, M. C.; SEN, A. (Ed.).The quality of life. Oxford: Clarendon Press, 1993. p. 30-54.

SERRA AZUL, Município [de]. Receitas e despesas do ensino - publicação 2010. Serra Azul, SP: Secretaria Municipal da Educação, 2014. Disponível em: <http://informacaomunicipal.com.br/serraazul/prefeitura/ trimestre10/quarto/_contas/trimestre.pdf>. Acesso em: 8 jul. 2015.

SILVA, R.; FERREIRA-LOPEZ, A. A Regional Development Index for Portugal. Social Indicators Research, V.118, n.3, p.1055-85, 2014.

SLAVOMIR, B. Title sustainable development in the world from the aspect of environmental health and Human Development Index: regional variations and patterns. Problemy Ekorozwoju, v. 11, n. 1, p. 11724, 2016.

SRINIVASAN, T. N. Human development: a new paradigm or reinvention of the wheel? American Economic Review, v. 84, n. 2, p. 238-43, 2012.

VALIAKHMETOV, R. M. Human development issues in the Republic of Bashkortostan. Sociological Studies, n. 8, p. 50-5, 2015.

VEIGA, J. E. Desenvolvimento sustentável: o desafio do século XXI. Rio de Janeiro: Garamond, 2008. A emergência socioambiental. São Paulo: Editora Senac, 2007. 


\section{Sobre os autores:}

André Cavalcante da Silva Batalhão: Pesquisador nível PhD Student no Center for Environmental and Sustainability Research, na Faculdade de Ciências e Tecnologia, Departamento de Ciências e Engenharia do Ambiente, da Universidade Nova de Lisboa. Doutorando em Ciências Ambientais na Universidade Federal de Goiás (CIAMB/UFG), Mestre em Desenvolvimento Regional e Meio Ambiente pela UNIARA (2013), Pós-graduado em Finanças e Banking pela Universidade Paulista (2010), Pós-graduado em Gestão e Manejo Ambiental na Agroindústria pela Universidade Federal de Lavras (2010). Trabalha com os seguintes temas: Indicadores de Sustentabilidade; Ferramentas de Avaliação de Sustentabilidade; Desenvolvimento Sustentável; Práticas e Ações Sustentáveis;Planejamento e Gestão Ambiental e da Sustentabilidade; Políticas Públicas; Gestão de Recursos Hídricos. E-mail: andre.ciamb.ufg@gmail.com

Glaucia Aparecida Prates: Graduação em Engenharia Florestal pela Universidade Federal de Viçosa (1986), mestrado em Engenharia Florestal pela Universidade Federal do Paraná (1990) e doutorado em Engenharia de Produção pela Universidade Federal de Santa Catarina (1998). Professora Assistente Doutor da Universidade Estadual Paulista Júlio de Mesquita Filho. Professora permanente do curso de pós-graduação- Mestrado profissional em Administração, na área de gestão de operações, no Campus de Jaboticabal- FCAV. E-mail: glaucia@itapeva.unesp.br

Denílson Teixeira: Possui graduação (licenciatura e bacharelado) em Ciências Biológicas pela Universidade Federal de São Carlos (UFSCar) (1989), especialização em Geoprocessamento na mesma instituição (2006), mestrado e doutorado em Ciências da Engenharia Ambiental pela Universidade de São Paulo (EESC/USP) (1993) e (2000) respectivamente. Atualmente é professor adjunto da Universidade Federal de Goiás (UFG), professor pesquisador do Programa de Pósgraduação em Engenharia Ambiental e Sanitária (PPEAS-UFG) e do Programa de Pós-Graduação em Ciências Ambientais (CIAMB-UFG). Tem desenvolvido sua pesquisa na área de Gestão Integrada de Recursos Hídricos; Indicadores Ambientais, Indicadores de Sustentabilidade e Educação. E-mail: dteixeira@ufg.br

Emiliano Lobo Godoi: Possui graduação em engenharia agronômica pela Universidade Federal de Viçosa (1988), mestrado e doutorado em Agronomia pela Universidade Federal de Goiás. Docente de nível superior da Universidade Federal de Goiás na Escola de Engenharia Civil. Experiência na área de gestão e planejamento ambiental, atuando principalmente nos seguintes temas: implantação de sistemas de gestão ambiental, gerenciamento ambiental de obras, controle ambiental de fontes poluidoras. E-mail: emiliano@ufg.br 